\title{
Análise comparativa do tratamento de lesões cutâneas dos membros inferiores com retalho sural versus retalho propeller*
}

\section{Comparative Analysis of the Treatment of Skin Lesions of the Lower Limbs with Sural Flap versus Propeller Flap}

\author{
Katherine V. Tenezaca1( ${ }^{1}$ Gustavo Bersani Silva10 Raquel Bernardelli lamaguchi1 ${ }^{10}$ \\ Marcelo Rosa de Rezende ${ }^{1(0)}$ Teng Hsiang Wei ${ }^{1(0)}$ Álvaro B. Cho ${ }^{10}$ \\ ${ }^{1}$ Grupo de Mão e Microcirurgia do Instituto de Ortopedia e \\ Traumatologia do Hospital das Clínicas da Faculdade de Medicina da \\ Universidade de São Paulo (IOT-HCFMUSP), São Paulo, SP, Brasil

\begin{abstract}
Endereço para correspondência Katherine V. Tenezaca, Residente do Grupo de Mão e Microcirurgia do Instituto de Ortopedia e Traumatologia do Hospital das Clínicas da IOT-HCFMUSP, Rua Ovídio Pires de Campos, 333, Cerqueira Cesar, São Paulo, SP, 05403-010, Brasil (e-mail: md.katherinetenezaca@gmail.com).
\end{abstract}

\section{Resumo}

Palavras-chave

- retalho perfurante

- retalhos cirúrgicos

- sitio doador de transplante

Abstract
Objetivo O objetivo do presente estudo foi comparar prospectivamente os retalhos sural e propeller para cobertura de partes moles da extremidade inferior. Foram avaliadas as seguintes variáveis: incidência de perda total ou parcial do retalho e morbidade da área doadora (fechamento primário versus enxerto de pele).

Métodos Análise prospectiva e randomizada de dados coletados de todos os pacientes apresentando defeitos em tecidos moles da extremidade distal da perna e do retropé submetidos aos retalhos em questão.

Resultados Foram avaliados 24 pacientes com idades entre 4 e 60 anos, entre 2011 e 2017. Cobertura completa foi obtida em 22 dos 24 pacientes $(91,6 \%)$ e observamos falha em 2 retalhos $(8,4 \%)$. O retalho sural, sendo a opção mais popular, continua a representar uma alternativa segura e versátil para defeitos cutâneos do terço distal da perna e da região do calcanhar. O retalho propeller, da mesma maneira, mostrou-se uma opção comparável para o tratamento destas lesões desafiadoras.

Conclusão Os retalhos sural e propeller são boas opções para a cobertura de partes moles da extremidade inferior, demostrando baixas taxas de complicações como perda parcial ou total do retalho.

Objective The objective of the present study was to prospectively compare the sural and propeller flaps for soft-tissues coverage of the lower extremity. The following

\footnotetext{
Trabalho desenvolvido no Grupo de Mão e Microcirurgia do Instituto de Ortopedia e Traumatologia do Hospital das Clínicas da Faculdade de Medicina da Universidade de São Paulo, São Paulo, SP, Brasil.
}

recebido

18 de Março de 2021

aceito

13 de Agosto de 2021

Publicado on-line

Fevereiro 9, 2022
DOI https://doi.org/ 10.1055/s-0041-1740199. ISSN 0102-3616. (c) 2022. Sociedade Brasileira de Ortopedia e Traumatologia. All rights reserved.

This is an open access article published by Thieme under the terms of the Creative Commons Attribution-NonDerivative-NonCommercial-License, permitting copying and reproduction so long as the original work is given appropriate credit. Contents may not be used for commercial purposes, or adapted, remixed, transformed or built upon. (https://creativecommons.org/ licenses/by-nc-nd/4.0/)

Thieme Revinter Publicações Ltda., Rua do Matoso 170, Rio de Janeiro, RJ, CEP 20270-135, Brazil 


\section{Keywords}

- perforator flap

- surgical flaps

- transplant donor site variables were evaluated: incidence of complete or partial flap loss and donor area morbidity (primary closure versus skin graft).

Methods Prospective and randomized analysis of data collected from all patients presenting with soft tissue defects of the lower third of the leg and heel treated with reverse sural or propeller flaps.

Results Twenty-four patients aged between 4 and 60 years old were evaluated between 2011 and 2017. Complete coverage was obtained in 22 of the 24 patients (91.6\%). Two flaps failed (8.4\%). The sural flap, being the most popular option, continues to represent a safe and versatile alternative for skin defects of the lower third of the leg and heel region. Likewise, the propeller flap was a comparable option to treat these challenging defects.

Conclusion Sural and propeller flaps are good options for soft tissues coverage of the lower extremity, with low complication rates (partial or total flap loss).

\section{Introdução}

O tratamento de lesões cutâneas dos membros inferiores é assunto de interesse não só pela elevada frequência com que se apresentam, mas principalmente pela dificuldade que impõe aos ortopedistas e cirurgiões plásticos. ${ }^{1-3}$ Especialmente complexas são as feridas que se localizam entre o terço distal da perna e o retropé, ${ }^{2,4}$ devido ao pequeno número de retalhos locais passíveis de serem utilizados para a cobertura desta região. ${ }^{5}$ Além disso, frequentemente, tais feridas são consequência de traumas de alta energia (notadamente acidentes motociclísticos) que acarretam lesões extensas de natureza grave. ${ }^{6,7}$ Classicamente, o retalho sural reverso é opção de tratamento, sendo que recentemente, com o desenvolvimento dos retalhos baseados no conceito de perfurantes cutâneas, o retalho em hélice ou propeller passou a ser uma ferramenta adicional no arsenal terapêutico para a cobertura de defeitos ao redor do tornozelo. ${ }^{8-12}$

Até o momento, não há estudos prospectivos comparando o retalho sural com o propeller no que se refere à taxa de sobrevida dos retalhos, à qualidade da cobertura e/ou à morbidade da área doadora, fato este que motivou a realização do presente estudo.

O objetivo do presente estudo foi comparar os retalhos sural e propeller, avaliando objetivamente, de modo prospectivo:

- A incidência de perda total do retalho

- A incidência de perda parcial do retalho

- A morbidade da área doadora: fechamento primário versus enxerto de pele.

\section{Casuística e Métodos}

O projeto de pesquisa foi registrado sob o número 1.551 .439 na plataforma Brasil. Os autores analisaram de maneira prospectiva e randomizada os dados coletados de pacientes que apresentavam defeitos de tecidos moles da perna ou do pé tratados pelo Grupo de Mão e Microcirurgia Reconstrutiva do Instituto de Traumatologia e Ortopedia do Hospital das Clínicas da Faculdade de Medicina da Universidade de São Paulo (IOT HC/FMUSP) entre 2011 e 2017. O critério de inclusão foi a realização de retalho fasciocutâneo (sural ou propeller) para cobertura da metade distal da perna e/ou do retropé, independentemente da idade. Pacientes com perdas cutâneas em outras regiões foram excluídos, assim como aqueles em quem não haveria a opção de cobertura cutânea com um dos dois tipos de retalhos estudados.

O defeito secundário, originado pela mobilização do retalho sural ou propeller, foi fechado primariamente ou através

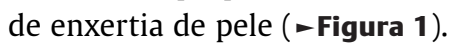

Foi incluído no presente estudo um total de 24 pacientes, 22 homens e 2 mulheres, com idade média de 37,7 anos ( 4 a

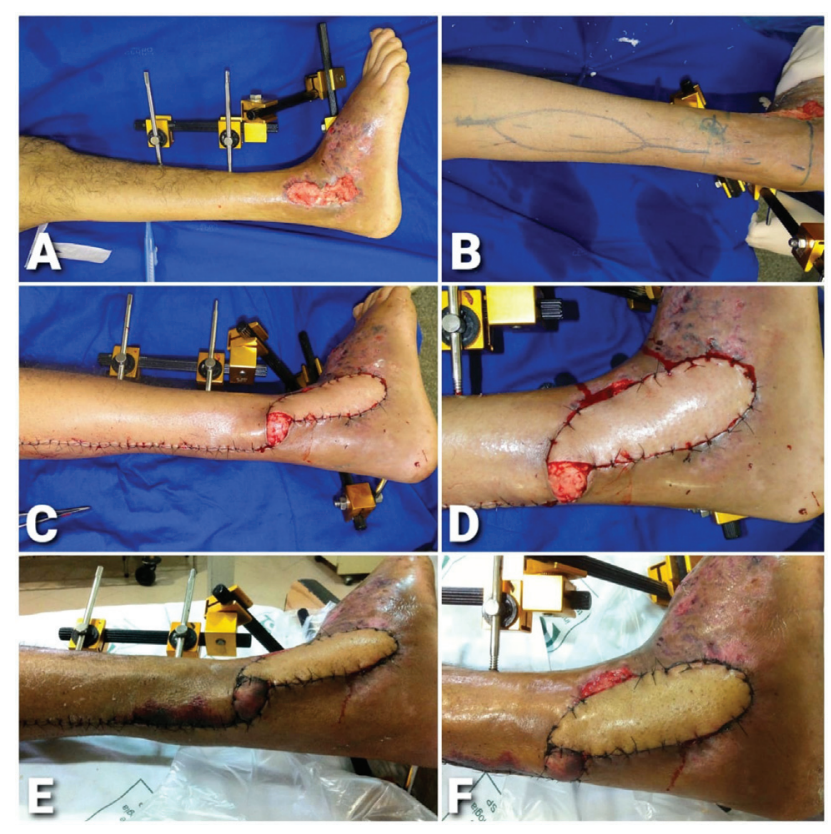

Fig. 1 (A e B) Defeito sobre o maléolo lateral. (B) Marcação do retalho sural. (C e D) Retalho dissecado, resultado pós-operatório. Fechamento primário da área doadora, com pequeno enxerto de pele parcial sobre o pedículo do retalho. (E e G) Pós-operatório (2 semanas). 


\begin{tabular}{|c|c|c|c|c|c|c|c|c|c|c|c|c|c|c|c|c|c|c|c|c|c|c|c|}
\hline 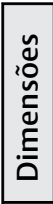 & \begin{tabular}{|c|c|} 
& \\
$b$ \\
$\times$ \\
$\dot{m}$ \\
\end{tabular} & $\begin{array}{l}E \\
\tilde{U} \\
\dot{x} \\
\times \\
\check{\sigma}\end{array}$ & 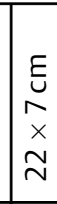 & \begin{tabular}{|c|}
$E$ \\
$E$ \\
$น$ \\
$\times$ \\
$\times$ \\
$O$ \\
\end{tabular} & \begin{tabular}{|c|}
$E$ \\
$\tilde{U}$ \\
0 \\
$\times$ \\
$\simeq$ \\
$\simeq$
\end{tabular} & $\begin{array}{c}E \\
\dot{x} \\
\times \\
\infty \\
\leftarrow\end{array}$ & $\begin{array}{l}E \\
E \\
b \\
\times \\
\sigma \\
\square\end{array}$ & $\begin{array}{c}E \\
\vdots \\
\infty \\
\times \\
\pm \\
\square\end{array}$ & $\begin{array}{l}E \\
E \\
6 \\
\times \\
0 \\
-\end{array}$ & $\begin{array}{c}E \\
\infty \\
\infty \\
\times \\
O \\
- \\
\end{array}$ & $\begin{array}{c}E \\
\text { U } \\
\times \\
\times \\
\llcorner \\
\square \\
\end{array}$ & 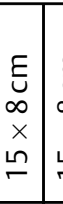 & 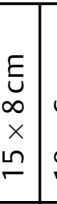 & $\begin{array}{l}E \\
E \\
b \\
\times \\
\sigma \\
-\end{array}$ & \begin{tabular}{l|l}
$\varepsilon$ & \\
$\vdots$ & \\
$b$ & \\
$\times$ & \\
$\sigma$ & \\
\end{tabular} & $\begin{array}{l}E \\
5 \\
\times \\
\times \\
0 \\
\end{array}$ & \begin{tabular}{l|l}
$E$ & \\
6 & \\
6 & \\
$\times$ & \\
0 & \\
0 & \\
\end{tabular} & 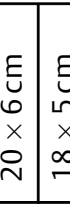 & 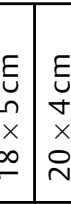 & $\begin{array}{l}E \\
\Xi \\
\forall \\
\times \\
\dot{ } \\
- \\
\end{array}$ & \begin{tabular}{|l}
$E$ \\
5 \\
0 \\
$x$ \\
0 \\
\end{tabular} & 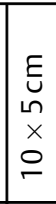 & 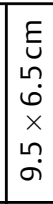 \\
\hline 可 & 岁 & है & 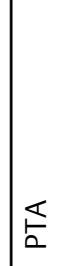 & 崩 & $\stackrel{\mathrm{L}}{\mathrm{L}}$ & $\mathbb{E}$ & 崩 & 峁 & 峁 & 崩 & $\stackrel{4}{<}$ & $\leftleftarrows$ & 崩 &  & 訔 & 这 & $\widehat{c} \mid$ & $\mathbb{E}$ & $\frac{\mathbb{a}}{a}$ & $\mathbb{E}$ & 文 & 崩 & है \\
\hline 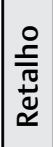 & 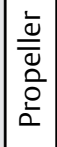 & $\tilde{n}$ & 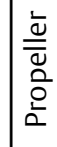 & $\sqrt{\frac{\pi}{5}}$ & 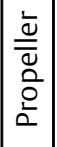 & 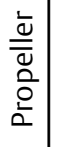 & $\begin{array}{l}\overline{0} \\
\overline{5}\end{array}$ & $\overline{\frac{\pi}{5}}$ & $\begin{array}{l}\bar{\pi} \\
\bar{\Xi} \\
\tilde{n}\end{array}$ & \begin{tabular}{|l}
$\bar{\pi}$ \\
$\bar{n}$
\end{tabular} & 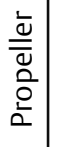 & 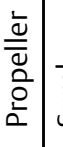 & $\sqrt{\frac{\pi}{3}}$ & 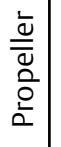 & 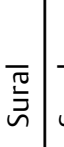 & $\overline{\widetilde{T}}$ & $\overline{\widetilde{T}}$ &  & 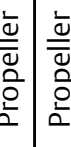 & 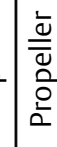 & $\overline{\stackrel{\widetilde{v}}{\leftrightarrows}}$ & $\overline{\bar{\pi}}$ & $\overline{\frac{\pi}{3}}$ \\
\hline
\end{tabular}


Tabela 2 Comparação entre os pacientes dos grupos Sural e Propeller

\begin{tabular}{|c|c|c|}
\hline & Grupo Sural & $\begin{array}{l}\text { Grupo } \\
\text { Propeller }\end{array}$ \\
\hline \multicolumn{3}{|l|}{ Pacientes ( $n^{\circ}$ de casos) } \\
\hline Homens & 12 & 10 \\
\hline Mulheres & 1 & 1 \\
\hline \multicolumn{3}{|l|}{ Idade (anos) } \\
\hline Média estatística $\pm \mathrm{DP}$ & $38.30 \pm 5.63$ & $35.25 \pm 3.89$ \\
\hline Máximo & 60 & 59 \\
\hline Mínimo & 04 & 17 \\
\hline \multicolumn{3}{|c|}{ Doença primaria ( $n^{\circ}$ de casos) } \\
\hline Lesão pós-traumática & 11 & 9 \\
\hline $\begin{array}{l}\text { Complicação } \\
\text { pôs-cirúrgica: deiscência }\end{array}$ & 0 & 1 \\
\hline Úlcera crônica & 1 & 1 \\
\hline Infecção crônica & 1 & 0 \\
\hline \multicolumn{3}{|c|}{ Localização para a reconstrução ( $n^{\circ}$ de casos) } \\
\hline Maleolar & 8 & 7 \\
\hline Tornozelo & 2 & 4 \\
\hline Calcanhar e dorso do pé & 3 & 1 \\
\hline
\end{tabular}

Abreviação: DP, desvio padrão.

60 anos). As características dos pacientes e a etiologia das lesões estão resumidas na - Tabela 1.

Todos os procedimentos foram realizados em uma única instituição pública, sendo os pacientes oriundos do ambulatório ou do pronto-socorro. Em todos os casos, a coleta de dados se realizou através do protocolo preenchido pelos autores do estudo (Anexo 1, disponível apenas on-line).

Os pacientes foram divididos em dois grupos conforme o retalho selecionado para a cobertura cutânea: Grupo Sural e Grupo Propeller (-Tabela 2). Os pacientes foram randomizados através de sorteio quando a cobertura cutânea do membro acometido era indicada. Desta maneira, 13 pacientes foram incluídos no Grupo Sural e 11 pacientes foram incluídos no Grupo Propeller, sendo 3 deles baseados em perfurantes da artéria fibular $(n=3)$ e 8 baseados em perfurantes da artéria tibial posterior $(n=8)$.

0 retalho sural foi delineado na região posterior-proximal da perna com seu ponto pivot demarcado $5 \mathrm{~cm}$ proximalmente à extremidade do maléolo lateral. A circulação sanguínea do retalho se deu por ramos venocutâneos e neurocutâneos oriundos dos vasos que acompanham o nervo sural e a veia safena parva, cuja irrigação arterial, por sua vez, se conecta ao sistema da artéria fibular. Sua dissecção foi realizada conforme a descrição clássica de Masquelet et al. ${ }^{13}$ (- Figura 2).

O retalho propeller foi de dois tipos, dependendo da localização do defeito cutâneo:

- Elevado no aspecto medial da perna, baseado em perfurantes da artéria tibial posterior;
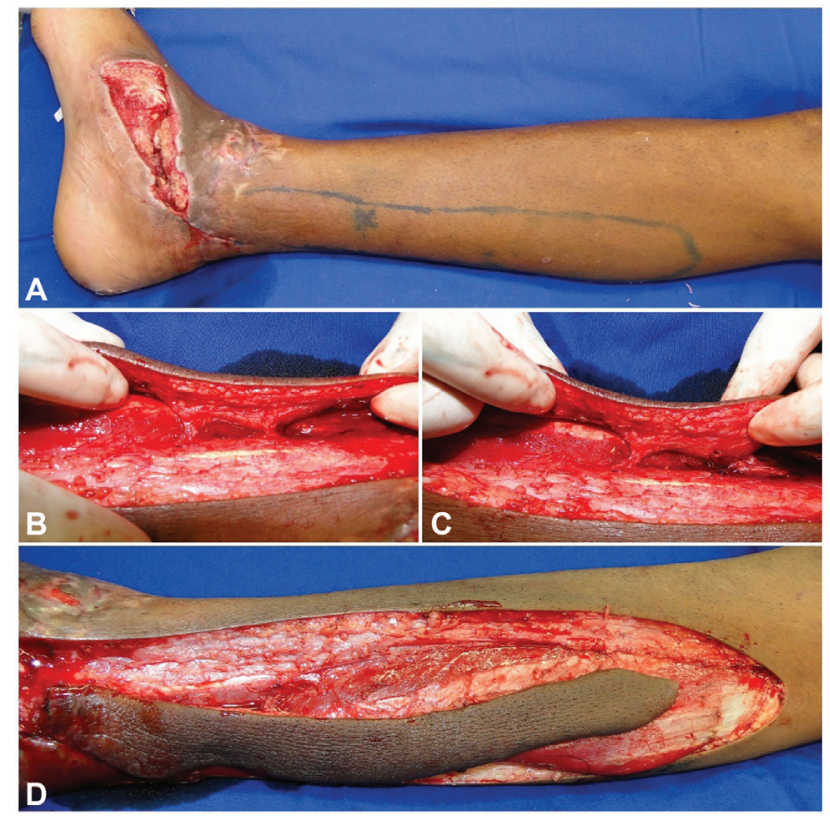

Fig. 2 (A) Paciente do sexo masculino, 31 anos, com defeito de partes moles sobre o maléolo medial $(8 \times 5 \mathrm{~cm})$. Marcação do retalho propeller medial [PM]. (B) Retalho dissecado (perfurantes da artéria tibial posterior). (C) Escolha de uma artéria perfurante como eixo principal do retalho. (D) Retalho dissecado.
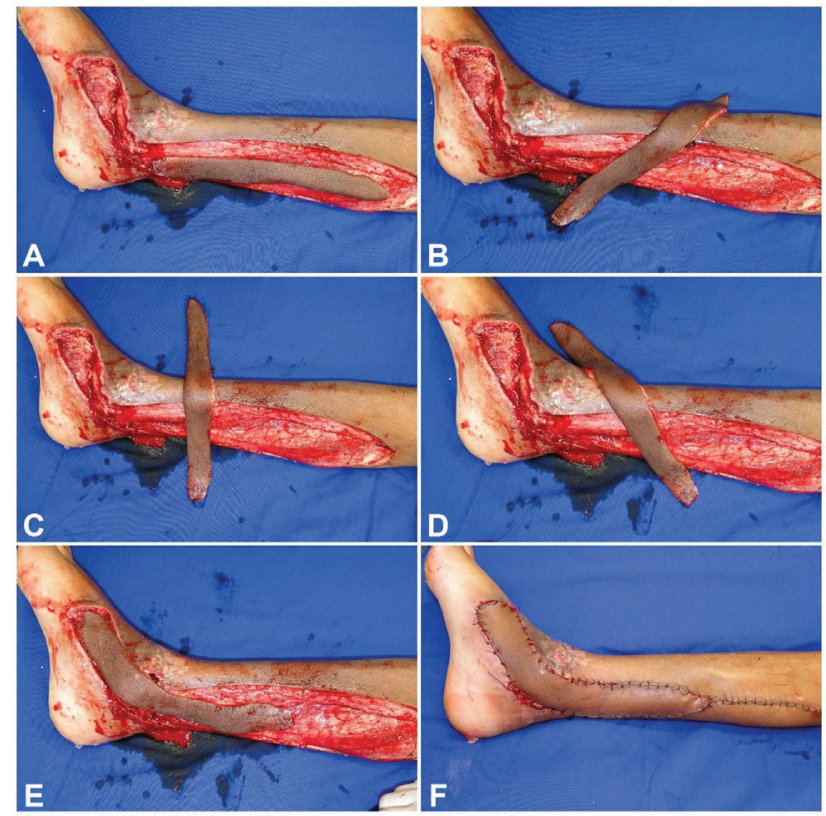

Fig. 3 (A-E) Sequência de rotação do retalho propeller medial [PM], de aproximadamente $180^{\circ}$ no sentido anti-horário, para cobertura do defeito. (F) Aspecto final, com fechamento primário da área doadora.

- Elevado no aspecto lateral da perna, baseado em perfurantes da artéria fibular.

A utilização de ultrassonografia com doppler para localização das perfurantes foi opcional e realizada em $45 \%$ dos pacientes $(n=5)$ do Grupo Propeller (-Figura 3 ). 
Tabela 3 Comparação entre os retalhos dos grupos Sural e Propeller

\begin{tabular}{|c|c|c|c|}
\hline & Grupo Sural & Grupo Propeller & \\
\hline \multicolumn{3}{|c|}{ Dimensões do retalho (média \pm DP; faixa) } & teste-t \\
\hline Comprimento $(\mathrm{cm})$ & $11.35 \pm 2.8 ; 19.0-9.0$ & $16.91 \pm 3.2 ; 20.0-12.0$ & $p=0.0002$ \\
\hline Largura (cm) & $6.2 \pm 1.2 ; 8.0-4.0$ & $5.8 \pm 1.2 ; 8.0-4.0$ & $\mathrm{p}=0.38$ \\
\hline Área $\left(\mathrm{cm}^{2}\right)$ & $71.98 \pm 26.3 ; 120-44$ & $98.64 \pm 29.8 ; 154-56$ & $\mathrm{p}=0.02$ \\
\hline \multicolumn{3}{|c|}{ Fechamento da área doadora ( $\mathrm{n}^{\circ}$ de casos [\%]) } & Teste de qui-quadrado \\
\hline Primário & $10(41.67 \%)$ & $6(25.0 \%)$ & $p=0.17$ \\
\hline Enxerto & $3(12.50 \%)$ & $5(20.8 \%)$ & $p=0.34$ \\
\hline \multicolumn{4}{|c|}{ Complicações pós-cirúrgicas ( ${ }^{\circ}$ de casos [\%]) } \\
\hline Infecção & $1(4.10 \%)$ & $2(8.30 \%)$ & $\mathrm{p}=0.57$ \\
\hline Necrose parcial & $2(8.30 \%)$ & $3(12.50 \%)$ & $p=0.62$ \\
\hline Necrose total & $2(8.30 \%)$ & $0(0 \%)$ & $p=0.24$ \\
\hline \multicolumn{4}{|c|}{ Cirurgia adicional (No. de casos) } \\
\hline Enxerto & 1 & 2 & \\
\hline Retalho novo & 3 & 1 & \\
\hline Reposicionamento & 0 & 1 & \\
\hline
\end{tabular}

Abreviação: DP, desvio padrão.

Nos dois grupos, as seguintes variáveis foram registradas: idade, etiologia, tamanho e localização do defeito, taxa de sobrevida do retalho, complicações pós-operatórias e cirurgias de revisão secundária. A análise estatística foi realizada através do IBM SPSS Statistics for Windows versão 25 (IBM Corp., Armonk, NY, EUA). O estado vascular do membro inferior envolvido foi avaliado clinicamente pelo estado da perfusão, pelo tempo de enchimento capilar e pela palpação dos pulsos.

\section{Análise Estatística}

Todos os dados foram apresentados como média mais desvio padrão (DP), procurando resumir as características dos pacientes e dos dois grupos de retalhos. As dimensões dos retalhos foram comparadas entre os dois grupos usando o teste $\mathrm{t}$ de Student. $\mathrm{O}$ fechamento da área doadora e as complicações foram analisadas pelo teste de qui-quadrado. O software GraphPad Prism (GraphPad Software, San Diego, CA, EUA) foi usado para a análise estatística, e valores$\mathrm{p}<0.05$ foram considerados como estatisticamente significativos (- Tabela 3 ).

\section{Resultados}

No Grupo Sural, a dimensão média do retalho foi de $14 \times 6 \mathrm{~cm}$, a taxa de perda parcial foi de $15 \%(2 / 13)$ e taxa de perda total foi de $15 \%$ (2/13). Houve necessidade de cirurgia adicional para desbridamento em quatro pacientes e de cobertura cutânea adicional com enxerto de pele em um paciente ou com novo retalho em três pacientes. A área doadora foi fechada primariamente em 10 pacientes, sendo que em 3 pacientes foi necessária enxertia de pele.

No Grupo Propeller, a dimensão média do retalho foi de $18 \times 6 \mathrm{~cm}$, a taxa de perda parcial foi de $27 \%(3 / 11)$, e a taxa de perda total foi de $0 \%$. Houve necessidade de cirurgia adicional para desbridamento em quatro pacientes e de cobertura cutânea adicional com enxerto de pele em dois pacientes ou com novo retalho em um paciente (perda parcial do propeller). A área doadora foi fechada primariamente em seis pacientes, sendo que em cinco pacientes foi necessária enxertia de pele (- Figura 4 ).

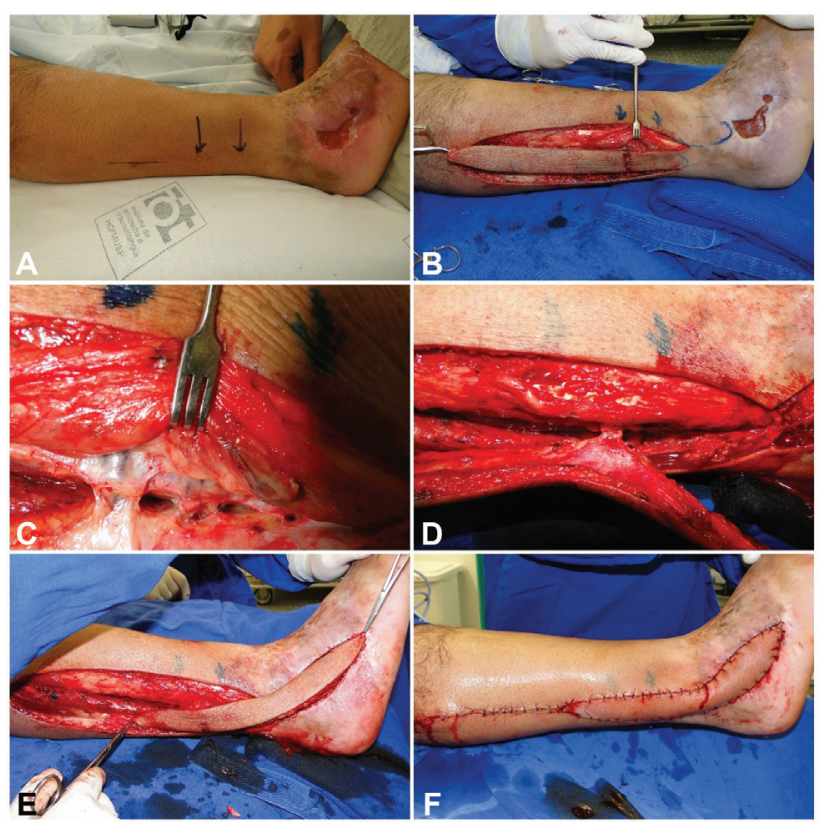

Fig. 4 (A) Defeito de tecidos moles sobre o maléolo lateral $(7 \times 4 \mathrm{~cm})$. (B) Marcação do retalho propeller lateral [PL]. (C) Retalho baseado em perfurantes da artéria fibular. (D) Ligadura de artéria perfurante secundária. (E) Rotação do retalho propeller lateral [PL] de aproximadamente $180^{\circ}$ no sentido horário. (F) Aspecto final, fechamento primário da área doadora. 
Tabela 4 Intercorrências de acordo com o tipo de retalho

\begin{tabular}{|c|c|c|c|c|c|c|c|}
\hline & & \multicolumn{5}{|c|}{ Intercorrências } & \multirow[t]{2}{*}{ Total } \\
\hline & & Nenhuma & Infecção & Perda parcial & Reposicionamento & Perda total & \\
\hline \multirow[t]{2}{*}{ Retalho } & Sural & 9 & 1 & 1 & 0 & 2 & 13 \\
\hline & Propeller & 7 & 2 & 1 & 1 & 0 & 11 \\
\hline \multicolumn{2}{|l|}{ Total } & 16 & 3 & 2 & 1 & 2 & 24 \\
\hline
\end{tabular}

Tabela 5 Fechamento da área doadora de acordo com o tipo de retalho

\begin{tabular}{|l|l|l|l|l|}
\hline \multicolumn{2}{|c|}{} & \multicolumn{2}{|l|}{$\begin{array}{l}\text { Fechamento da área } \\
\text { doadora }\end{array}$} & \multirow{2}{*}{ Total } \\
\cline { 2 - 4 } \multicolumn{2}{|c|}{} & Primário & Enxerto & \\
\hline \multirow{2}{*}{ Retalho } & Sural & 10 & 3 & 13 \\
\cline { 2 - 5 } & Propeller & 6 & 5 & 11 \\
\hline \multicolumn{2}{|l|}{ Total } & 16 & 8 & 24 \\
\hline
\end{tabular}

Considerando o grupo total de pacientes (Grupos Sural e Propeller), não foram encontradas diferenças significativas nas incidências de perda parcial e total dos retalhos e a cobertura cutânea completa foi obtida em 22 dos 24 pacientes (91.6\%). Dois retalhos (Grupo Sural) apresentaram falha evoluindo para perda total (15\%; 2/13 Grupo Sural).

Em 22 pacientes cujos retalhos evoluíram favoravelmente, 16 não apresentaram nenhum tipo de complicação (66\%). Três desenvolveram infecção (12.5\%), 2 com necrose da borda distal com perda parcial do retalho (8.3\%) e 1 paciente $(4.1 \%$; Grupo Propeller) apresentou comprometimento da perfusão do retalho após sua mobilização para a área receptora, sendo necessário o reposicionamento deste ao leito de origem para autonomização e mobilização final após 1 semana, logrando finalmente êxito na cobertura da lesão ( - Tabela 4).

Três pacientes (2 Propeller, 1 Sural) necessitaram ainda de reposicionamento e de coberturas com enxerto de pele em áreas cruentas residuais em associação aos retalhos.

A análise comparativa dos retalhos sural e propeller não demonstrou diferença quanto à morbilidade da área doadora. O fechamento primário da área doadora foi realizado em $67 \%$ dos pacientes $(16 / 24)(p=0.17)$, sendo necessário enxerto de pele parcial em 33\% (8/24) $(p=0.34)$ (-Tabela 5). Foi possível fechamento primário em $76 \%$ dos pacientes do Grupo Sural; já no Grupo Propeller, o fechamento foi possível em $55 \%$ dos pacientes.

\section{Discussão}

O objetivo da cirurgia reconstrutiva do membro inferior é a obtenção de membros funcional e esteticamente adequados. A complexidade da reconstrução depende, dentre outros fatores, da energia e do mecanismo de trauma, da irrigação do membro e das comorbidades de cada paciente. ${ }^{14,15}$ As opções são: retalhos regionais únicos (perfurantes ou neurocutâneos, por exemplo), múltiplos retalhos combinados e retalhos microcirúrgicos. ${ }^{16}$
Os retalhos regionais têm como benefícios a menor complexidade em sua elevação (prescindindo de técnica microcirúrgica), utilização de tecidos do próprio membro lesado, menor tempo cirúrgico e preservação dos eixos vasculares dos membros inferiores. Retalhos regionais fasciocutâneos oferecem uma forma segura e versátil para coberturas no segmento distal da perna e no retropé. ${ }^{15,16}$

O retalho sural reverso, identificado pela primeira vez por Taylor et al. ${ }^{17}$ em 1975 , é o retalho regional de padrão axial mais utilizado para a região distal da perna e o retropé. ${ }^{18}$ Ele foi popularizado por Masquelet et al. ${ }^{13}$ em 1992, que confirmaram o suprimento arterial retrógrado, a sua relação com o nervo sural e sua drenagem venosa.

A introdução dos retalhos em hélice ou tipo propeller expandiu as opções para a cobertura cutânea dos membros inferiores. Descrito pela primeira vez por Hyakusoku et al., ${ }^{19}$ o retalho propeller pode ser desenhado em qualquer local onde exista uma perfurante presente. Além disso, a morbidade do local doador pode ser mínima, sendo geralmente possível o fechamento primário. ${ }^{19}$ A técnica operatória tem sido gradualmente aperfeiçoada nos últimos anos, de modo que, atualmente, os retalhos do tipo propeller são considerados seguros e eficazes. ${ }^{3}$

A região posterior da perna é suprida pelo angiossoma sural, baseado nas artérias surais musculocutâneas: artérias surais superficiais média, mediana e lateral, suprindo a pele e a fáscia desta região. A artéria superficial mediana é a maior, cursando proximalmente desde a fossa poplítea e seguindo entre as duas cabeças dos músculos gastrocnêmios (rafe). Proximalmente, seu trajeto é subfascial (mais profundo) e, distalmente, se torna subdérmico ao nível da união musculotendinosa do músculo gastrocnêmio lateral. No tornozelo, esta arteríola é acompanhada pelo nervo sural e encontra-se medial à veia safena parva ao nível do maléolo lateral. Ela mantém numerosas anastomoses com a artéria fibular ao longo do seu curso, vasos que serão ligados e divididos durante a dissecção para a mobilização do retalho sural reverso. Distalmente, a uma distância de entre 5 e $6 \mathrm{~cm}$ proximais da ponta do maléolo lateral, está localizado o ponto pivot potencialmente mais distal do pedículo, que permite uma maior amplitude de rotação do retalho. ${ }^{20}$

Atualmente, existem poucos estudos comparando o retalho sural versus o propeller. Demiri et al. ${ }^{15}$ publicaram um estudo comparativo retrospectivo sobre os retalhos neurocutâneos reversos versus perfurantes do tipo hélice (propeller) para a reconstrução do pé diabético, obtendo altas taxas de sucesso (entre 95 e 97\%) com ambos os retalhos. Os resultados do presente estudo evidenciam, igualmente, altas taxas de 
sucesso no que se refere à cobertura cutânea, com baixas taxas de complicações (perda parcial ou total). $O$ retalho sural, sendo mais utilizado, segue representando uma alternativa segura e versátil para defeitos cutâneos do terço distal da perna e do tendão calcâneo. Os retalhos propeller baseados em perfurantes da artéria fibular ou tibial posterior também foram opções viáveis para a cobertura cutânea desta região. Quando não foi possível um fechamento primário na área doadora, o enxerto de pele parcial foi utilizado na presente série com resultados funcionais e estéticos adequados.

Mesmo com o pequeno número de pacientes estudados, acreditamos que a análise prospectiva e randomizada das técnicas contribui para a tomada de decisão dos cirurgiões reconstrutivos.

\section{Conclusão}

Os retalhos sural e propeller mostraram-se opções viáveis para o tratamento de lesões de partes moles do terço inferior da perna e do retropé. A comparação prospectiva e randomizada entre as técnicas evidenciou baixas taxas de perda parcial ou total dos retalhos, assim como de complicações.

\section{Fontes de Financiamento}

A presente pesquisa não recebeu nenhuma bolsa específica de agências de fomento nos setores público, comercial ou sem fins lucrativos.

Conflito de Interesses

Os autores declaram não haver conflito de interesses.

\section{Referências}

1 Zhang F, Lin S, Song Y, Zhang G, Zheng H. Distally based sural neuro-lesser saphenous veno-fasciocutaneous compound flap with a low rotation point: microdissection and clinical application. Ann Plast Surg 2009;62(04):395-404

2 Aoki S, Tanuma K, Iwakiri I, et al. Clinical and vascular anatomical study of distally based sural flap. Ann Plast Surg 2008;61(01):73-78

3 Jakubietz RG, Jakubietz MG, Gruenert JG, Kloss DF. The 180degree perforator-based propeller flap for soft tissue coverage of the distal, lower extremity: a new method to achieve reliable coverage of the distal lower extremity with a local, fasciocutaneous perforator flap. Ann Plast Surg 2007;59(06):667-671

4 Tos P, Innocenti M, Artiaco S, et al. Perforator-based propeller flaps treating loss of substance in the lower limb. J Orthop Traumatol 2011;12(02):93-99
5 Noack N, Hartmann B, Küntscher MV, Ku MV. Measures to prevent complications of distally based neurovascular sural flaps. Ann Plast Surg 2006;57(01):37-40

6 Moscatiello F, Masià J, Carrera A, Clavero JA, Larrañaga JR, Pons G. The 'propeller' distal anteromedial thigh perforator flap. Anatomic study and clinical applications. J Plast Reconstr Aesthet Surg 2007;60(12):1323-1330

7 Costa-Ferreira A, Reis J, Pinho C, Martins A, Amarante J. The distally based island superficial sural artery flap: clinical experience with 36 flaps. Ann Plast Surg 2001;46(03):308-313

8 Rajendra Prasad JS, Cunha-Gomes D, Chaudhari C, Bhathena HM, Desai S, Kavarana NM. The venoneuroadipofascial pedicled distally based sural island myofasciocutaneous and muscle flaps: anatomical basis of a new concept. Br J Plast Surg 2002;55(03): 203-209

9 Murakami M, Hyakusoku H, Ogawa R. The multilobed propeller flap method. Plast Reconstr Surg 2005;116(02):599-604

10 Lu TC, Lin CH, Lin CH, Lin YT, Chen RF, Wei FC. Versatility of the pedicled peroneal artery perforator flaps for soft-tissue coverage of the lower leg and foot defects. J Plast Reconstr Aesthet Surg 2011;64(03):386-393

11 Pignatti M, Ogawa R, Hallock GG, et al. The "Tokyo" consensus on propeller flaps. Plast Reconstr Surg 2011;127(02):716-722

12 Panse NS, Bhatt YC, Tandale MS. What is safe limit of the perforator flap in lower extremity reconstruction? Do we have answers yet?. Plast Surg Int 2011;2011:349357

13 Masquelet AC, Romana MC, Wolf G. Skin island flaps supplied by the vascular axis of the sensitive superficial nerves: anatomic study and clinical experience in the leg. Plast Reconstr Surg 1992; 89(06):1115-1121

14 Akhtar S, Ahmad I, Khan AH, Khurram MF. Modalities of softtissue coverage in diabetic foot ulcers. Adv Skin Wound Care 2015;28(04):157-162

15 Demiri E, Tsimponis A, Pavlidis L, Spyropoulou GA, Foroglou P, Dionyssiou D. Reverse neurocutaneous vs propeller perforator flaps in diabetic foot reconstruction. Injury 2020;51(Suppl 4): S16-S21

16 Ibrahim A, Oneisi A. Lower Extremity Reconstruction. In: AbuSittah G, Hoballah J, Bakhach J, editors. Reconstructing the War Injured Patient. Cham, Switzerland: Springer Internacional Publishing; 2017:79-88

17 Taylor GI, Daniel RK. The anatomy of several free flap donor sites. Plast Reconstr Surg 1975;56(03):243-253

18 Donski PK, Fogdestam I. Distally based fasciocutaneous flap from the sural region. A preliminary report. Scand J Plast Reconstr Surg 1983;17(03):191-196

19 Hyakusoku H, YamamotoT, Fumiiri M. The propeller flap method. Br J Plast Surg 1991;44(01):53-54

20 Cho AB, Pohl PH, Ruggiero GM, Aita MA, Mattar TG, Fukushima WY. The proximally designed sural flap based on the accompanying artery of the lesser saphenous vein. J Reconstr Microsurg 2010;26(08):501-508 